\title{
OTIMIZAÇÃO DA ETAPA DE NEUTRALIZAÇÃO, DO PROCESSO DE GALVANIZAÇÃO ELETROLÍTICA, UTILIZANDO A MODELAGEM ARIMA
}

\author{
Flávio Roberto Andara ${ }^{1}$; Adriano Mendonça Souza ${ }^{2}$ \\ ${ }^{1}$ Programa de Pós-Graduação em Engenharia de Produção - PPGEP \\ Universidade Federal de Santa Maria - UFSM- Santa Maria/RS - Brasil \\ Flavio.andara@ibiruba.ifrs.edu.br
}

2Professor Titular do Programa de Pós-Graduação em Engenharia de Produção - PPGEP

Área de concentração- Métodos Quantitativos para a Tomada de Decisão

Universidade Federal de Santa Maria - UFSM - Santa Maria/RS - Brasil

Amsouza.sm@gmail.com

Resumo

Muitos processos produtivos atuais apresentam uma dinâmica que os classifica, dentro de uma ótica de controle estatístico, de processos multivariados onde apresentam correlação e autocorrelação entre os dados amostrais. Dentro dessa realidade são avaliadas duas etapas, de enxágüe e neutralização, do processo de galvanização eletrolítica, processo químico que configura um dos melhores exemplos de produção de observações não independentes. Os dados utilizados na pesquisa foram extraídos do processo em questão em uma empresa que atua no segmento de processos de usinagem. Foram coletadas 125 observações do processo de galvanização eletrolítica, no mês de outubro de 2013. Os dados reais do processo galvânico foram trabalhados via metodologia de séries temporais, utilizando os modelos Auto-regressivo Integrados de Médias Móveis - ARIMA, por meio da metodologia de Box e Jenkis, onde ao final da avaliação teremos uma estruturação gráfica realizada com os resíduos encontrados do melhor modelo e sobre esses será feita uma avaliação do processo em questão. O trabalho estatístico foi desenvolvido com o uso do Pacote Econométrico Eviews, versão 8.

Palavras-chave: galvanização eletrolítica; processo multivariado; autocorrelação.

Abstract

A lot of productive current processes present dynamic that classify them into a statistic's control, in multivariate processes, where they present correlation and autocorrelation among sample datas. In this reality, two stages are evaluated: rinse and neutralization, in electrolytic galvanizing process, a chemistry process that configures one of the best non-independent views production examples. The datas used in the research were extractedin this process in an enterprise which acts in machining process segment. One hundred twenty five observations in electrolytic galvanizing process were collected on October, $31^{\text {st }}$, 2013. The real datas of galvanic process were worked through temporal series' methodology using the average-moving Integrated auto-regressive models - ARIMA, through of Box and Jenkis methodology, where the final assessment will have a structuring graphic performed with the best model residues found and about these will be done a process evaluation. The statistic work was developed using Eviews Econometric package, version eight.

Key word: electrolytic galvanizing; multivariate process; autocorrelation. 


\section{INTRODUÇÃO}

Existem inúmeras situações nas quais a qualidade de um produto é determinada por um somatório de etapas bem sucedidas de um processo. A operação de galvanização eletrolítica não foge a essa regra, e, como tal, apresenta uma quantidade considerável de etapas que, ao se somarem, resultam em um produto galvanizado, com suas características estéticas e, principalmente, de resistência à corrosão aumentada. Segundo Zempulski (2007), na galvanização eletrolítica a proteção pelo uso do zinco consiste em combinar o zinco com o ferro, resultando no zinco como ânodo e o ferro como cátodo. Prevenindo assim a corrosão do ferro, dado que o zinco sofrerá a corrosão antes do ferro.

As empresas buscam, para continuarem mantendo seus lucros, frente a mercados com exigências cada vez maiores, controles mais efetivos no uso total de suas matérias-primas. Desta forma busca ter um conhecimento real do quanto cada insumo pode contribuir na etapa em que será envolvido e o quanto ele pode contribuir no processo, sendo necessária a identificação de seu impacto no processo de modo que seus efeitos possam ser avaliados, para que uma ação possa ser tomada.

No processo de galvanização, caracterizado por diversos banhos químicos e de enxágüe, é possível realizar correções e ajustes de forma a minimizar os custos de reciclagem dos efluentes envolvidos que é um problema que não se pode desconsiderar, tanto do ponto de vista econômico como de responsabilidade ambiental para a empresa, sendo necessário que a qualidade dos banhos de enxágue sejam mantidos para garantir produtos finais de qualidade.

A pesquisa apresentada é inicial e faz parte de um conjunto de investigações e trabalhos relacionados ao controle de qualidade e a modelagem estatística, que visam justificar o uso dos modelos de séries temporais como alternativa para um controle efetivo de processos autocorrelacionados.

Portanto, o objetivo da pesquisa é monitorar o processo de galvanização eletrolítica via cartas de controle de resíduos, os quais são oriundos da modelagem ARIMA, dado que o processo em análise é um processo químico e apresenta-se autocorrelacionado.

\section{REVISÃO DE LITERATURA}

\subsection{Cartas de controle}

As primeiras cartas de controle utilizadas largamente na indústria de processo foram desenvolvidas por Walter A. Shewhart em 1932. Estas cartas são estruturas com uma linha central (LC), que representava o valor alvo do processo, por outras duas linhas, acima e abaixo dessa linha central, denominadas limite superior de controle (LSC) e limite inferior de controle (LIC). Essas cartas têm como principal função o monitoramento dos processos produtivos.

As cartas de controle, que visam o monitoramento dos processos produtivos, apresentam quatro ciclos que, de acordo com Cai et al(2002, p.86), são: Um estado de controle do processo produtivo; um estado fora de controle; um estado de avaliação de causas assinaláveis e, por fim, um estado de ajuste.

Outra análise importante sobre as cartas de controle refere-se aos seus custos, pois uma consideração lógica das dimensões de um processo considerado ótimo, feita por Mingoti (2008, p.55), considera a economia, a eficiência, a produtividade e a qualidade como primordiais.

Existem muitas vantagens na implementação das cartas de controle, como citadas por Pereira (2008, p. 350) que são: Prevenção de ocorrência de produto não conforme; distinção entre causas de variação comuns e especiais; facilidade de utilização das cartas pelo operador no seu posto de trabalho, consistência e previsão da qualidade e custos, menor custo por unidade produzida e utilização de uma linguagem comum. 
Nessa mesma linha de raciocínio os custos podem ser classificados em seis tipos, que, avaliados por Cai et al (2002, p.86), são: custo de amostragem; custo do processo quando este está rodando fora de controle; custo deste processo em controle; custo de falsos alarmes; custo de busca de causas assinaláveis quando o processo está fora de controle e o custo de ajuste.

Ainda, esta ferramenta de controle apresenta duas fases, como determinado por Woodall (2000, p.342), a fase um, que objetiva verificar se o processo está ou não em controle estatístico, e a fase dois, que tem como função determinar as variações de um processo em controle.

Na mesma direção, Souza, A. et al (2012), sugere cartas de controle especiais que captem a presença da correlação gerada entre as observações não independentes - gráficos de controle de resíduos. Ou cartas de controle para captar o efeito da correlação entre as variáveis para monitorar todas as características simultaneamente - gráficos multivariados. Se for observada a presença de correlações entre as seqüências de operações torna-se pertinente o controle na presença desta correlação entre os instantes de tempo de processamento. Sendo necessário utilizar gráficos de controle de resíduos.

Os gráficos de controle de resíduos de acordo com Rotondaro (2002), são aplicáveis para valores individuais e para a amplitude móvel, conforme as equações 3, 4 e 5.

$$
\begin{aligned}
& L S C_{X}=\bar{X}+E_{2} \cdot \bar{R} m \\
& L I C_{X}=\bar{X} \\
& L I C_{X}=\bar{X}-E_{2} \cdot \bar{R} m
\end{aligned}
$$

Já, para o gráfico da amplitude móvel são empregadas as equações 6, 7 e 8 mostradas abaixo.

$$
\begin{aligned}
L S C_{R m} & =D_{4} \cdot \bar{R}_{m} \\
L M_{R m} & =\bar{R} m \\
L I C_{R m} & =D_{3} \cdot \overline{R_{m}}
\end{aligned}
$$

Esta aplicação só é possível se as características a serem analisadas são independentes, seguem a distribuição Normal, isto é, independentes e identicamente distribuídas (i.i.d).

\subsection{Análise de séries temporais}

Para a avaliação das variáveis envolvidas no processo, serão utilizados os modelos ARIMA que, segundo Morettin (2006), é bastante utilizado na análise de processos autocorrelacionados. Essa modelagem permite captar o comportamento da correlação seriada ou autocorrrelação entre os valores da série temporal e, utilizá-los para se encontrar resíduos i.i.d. para serem analisados por gráficos de controle. Nesse processo altamente dinâmico, onde as observações não são independentes, é sugerido por Costa et al (2012), que estas não devam ser monitoradas por gráficos convencionais.

Conforme Lee (2010, p.67), os modelos ARIMA podem ser representados como a combinação das observações passadas e dos erros também passados. A estratégia para a construção do melhor modelo é baseada em um ciclo iterativo, que, segundo Morettin (2006), segue os seguintes passos: I- Especificação do modelo; II - Identificação de um modelo; III estimação do modelo e IV - verificação ou diagnóstico do modelo ajustado.

Assim, de acordo com Oliveira et al (2012, p.17), os modelos ARIMA (p, d, q) são modelos que sofreram uma ou duas diferenciações para tornarem-se estacionários, sendo que o d representa o número de diferenciações empreendidas para tornar a série estacionária.

Para concluir, os modelos ARIMA complementam-se por: 
- Modelo auto-regressivo de ordem $\mathrm{p}$ - $\mathrm{AR}(\mathrm{p})$ : usado quando há autocorrelação entre as observações, é descrito por seus valores passados regredidos e mais um ruído aleatório $\varepsilon_{\mathrm{t}}$;

- Modelo de média móvel de ordem $q$ - MA(q): resulta da combinação dos ruídos brancos $\varepsilon \varepsilon_{t}$ do período atual com aqueles ocorridos em períodos anteriores;

- Modelo auto-regressivo de média móvel - ARMA (p,q): usado quando há autocorrelação entre as observações passadas mais uma combinação dos ruídos brancos dos resíduos.

De acordo com Souza A. et al (2012, p.114), um processo não estacionário segue um modelo ARIMA (p, d, q) como na equação (1).

$$
\phi(B) \Delta^{d} X_{t}=\theta(B) u_{t}
$$

Sendo que o processo estacionário pode ser representado por um ARIMA (p,q), de acordo com a equação (2).

$$
\phi(B) X_{t}=\theta(B) u_{t}
$$

$\mathrm{Na}$ metodologia de Box-Jenkis, para poder estimar os parâmetros do modelo autoregressivo de médias móveis deve-se verificar se a série é estacionária, caso não seja, deve-se torná-la estacionária.

Com a estimação dos parâmetros através da função de Mínimos Quadrados Ordinários e, de acordo com Otero et al (2001, p.202), a técnica de análise de séries temporais é uma ferramenta útil, porém com sistemáticas para modelagem e interpretações de resultados nem sempre triviais.

Para a determinação do melhor modelo utiliza-se os critérios penalizadores, Akaike Information Criteria- AIC e Schwartz Bayesian Criteria - BIC, determinados pelas equações 9 e 10. Por fim a decisão também será baseada no critério da parcimônia, situação onde se encontra o menor número de parâmetros.

$$
\begin{aligned}
& A I C=-2 \operatorname{Ln}(L)+2 n \\
& B I C=-2 \operatorname{Ln}(L)+n \cdot \operatorname{Ln}(T)
\end{aligned}
$$

Encontrado o melhor modelo e interpretado os resultados, utiliza-se gráficos de controle aplicados sobre os resíduos dos melhores modelos encontrados, para verificar se o processo produtivo encontra-se estável, pois, estes resíduos, conforme utilizados por Casarin et al (2013, p.03), são úteis no monitoramento do desempenho das variáveis, desde que atendendo as suposições de não autocorrelacionados, média zero e variância constante.

\section{METODOLOGIA DA PESQUISA}

\subsection{Descrição do processo produtivo}

Montgomery (2009, p.03), afirma que a qualidade é inversamente proporcional a variabilidade, e, partindo dessa premissa, buscamos conhecer o processo de galvanização eletrolítica em todos os seus detalhes.

No processo de galvanização eletrolítica existem pelo menos 14 etapas que o subdivide, para o desenvolvimento da pesquisa optou-se pela análise das etapas de enxágüe e neutralização, que são operações que se realizam antes do processo de deposição galvânica propriamente. Vale observar que neste estudo busca-se a explicação da etapa de enxágue em função de seus valores passados, assim como na etapa de neutralização, caracterizando desta forma uma série temporal.

O processo de galvanização eletrolítica consiste em uma seqüência de banhos que envolvem basicamente três etapas: o pré-tratamento, o revestimento e a passivação. 
Durante o pré-tratamento é feito um banho de desengraxe, com soda cáustica, e, logo depois a decapagem, mergulhando-se em solução de ácido muriático para remover óxidos, tintas, ferrugem e incrustações da superfície metálica. Em seguida as peças passam por um enxágüe em água para evitar a contaminação dos banhos seguintes.

Depois da decapagem as peças passam por uma neutralização, a última etapa do prétratamento, cuja função é eliminar restos de líquidos e restos salinos dos poros, ou outras imperfeições. Nesta etapa utilizam-se líquidos com reações fracamente ácidas, ou alcalinas; neste caso específico se utiliza o banho de hidróxido de sódio, conhecido como soda cáustica.

Inicia-se então a etapa do processo de galvanização - o revestimento - e refere-se à deposição eletrolítica. Neste processo é usado o banho de zinco ( $\mathrm{Zn})$, onde as peças ficam submersas por alguns minutos. Esta etapa é responsável por aumentar a resistência à corrosão.

A peça passa novamente pelo enxágüe e segue para a passivação: banho contendo ácido nítrico que tem por finalidade fixar o zinco e dar um acabamento tipo espelhado à peça. Depois de passar por todas as essas etapas é feito novamente um enxágüe e em seguida as peças são encaminhadas para secagem. As peças maiores secam ao ar livre, já as peças pequenas secam em centrífugas para evitar que sequem coladas umas às outras.

\subsection{Etapas metodológicas}

No planejamento da pesquisa definiram-se as seguintes etapas:

- Coleta de um volume constante das soluções líquidas, nos respectivos tanques de processo, referentes às etapas de enxágüe e neutralização, totalizados 125 amostras, capturadas de cinco em cinco minutos durante o mês de outubro de 2013;

- Os registros, referentes ao enxágue são da condutividade, medido em micro siemens por centímetro $(\mu \mathrm{s} / \mathrm{cm})$, onde foi utilizado um condutivímetro, marca Quimis, modelo Q $405 \mathrm{M}$, com resolução milesimal. E os referentes à neutralização é o $\mathrm{Ph}$, medido com o pHmetro marca Sanxin, modelo PHS-3D ph meter, com resolução centesimal. Todos os instrumentos apresentam suas calibrações dentro do prazo de validade.

-Coletadas as informações necessárias das variáveis selecionadas, passou-se às análises preliminares para encontrar os modelos ARIMA que possam representar tais variáveis:

-Análise dos valores de correlação a autocorrelação parcial, por meio das funções de correlação e autocorrelação parcial, utilizados na forma de inspeção gráfica e úteis para sinalizar a ordem do modelo a ser utilizado;

- Investigar a estacionariedade da série por meio dos:

-Testes Augmented Dickey-Fuller (1984) - (ADF), que possuem como hipótese nula que a série é não estacionária e integrada de ordem $\mathrm{d}(\mathrm{d}>0)$. Na grande maioria dos casos de séries não estacionárias, basta uma diferenciação para tornar a série resultante estacionária, isto é, 1 (0). E, como uma forma de validação dos testes ADF, utiliza-se o teste Kwiatkowski-PhillipsSchmidt-Shin (1992) - (KPSS), em que as hipóteses apresentadas são inversas aos testes ADF, ou seja, H0 postula que a série é I(0) contra a alternativa que a série seja H1(1). Esses testes são amplamente discutidos na literatura de econometria, como por Enders (1995), Maddala (1992) e Souza, A. et al (2011);

-Determinado o grau de estacionariedade da série a ser estudada, utiliza-se a metodologia de Box-Jenkis descrita pelos autores em 1970 para determinação do modelo ARIMA adequado, de modo que gerem resíduos i.i.d. atingindo as imposições necessárias para a aplicação de gráficos de controle de resíduos.

- Determinado os resíduos, aplicam-se os gráficos de controle de resíduos, que de acordo com Rotondaro (2002), como forma de avaliar as variáveis envolvidas no processo produtivo. 
- Vencidas as etapas de modelagem e elaboração dos gráficos de controle, faz-se a análise para identificar pontos que podem causar anomalia no processo produtivo e quando necessário toma-se uma ação corretiva.

\section{ANÁLISE DOS RESULTADOS}

As séries em estudo apresentaram-se como não estacionárias, situações evidenciadas pelos gráficos das séries originais nas Fig. 1 e 2. Ainda, para confirmação da estacionariedade das séries utilizou-se os testes de raízes unitárias, ADF e o KPSS onde as mesmas foram classificadas como não estacionárias em nível, com 5\% de significância. Desta forma as duas séries serão tratadas como não estacionárias, realizando dessa forma, as estimativas possíveis. De acordo com Souza, A. et al( 2012, p.116), a condição de estacionariedade da série é importante para obter parâmetros estáveis e permitir a produção de diagnósticos precisos.

Fig. 1 - Gráf. da série original - var.enxágüe.

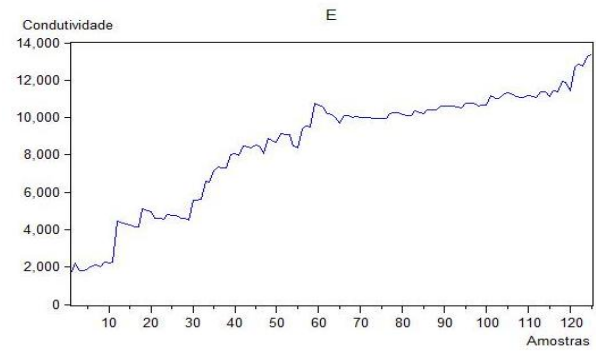

Fig. 2 - Gráf. da série original - var. neutralização

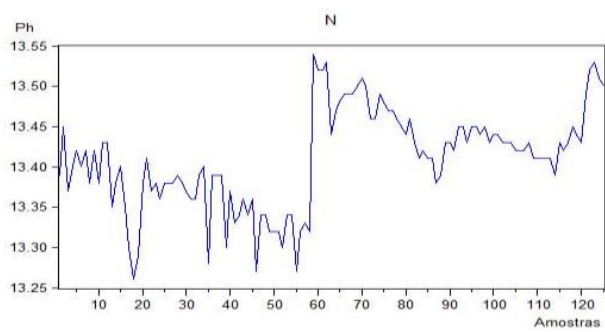

De acordo com as Fig. 1 e 2 observa-se que as séries são não estacionárias, portanto ao ajustar o modelo ARIMA, as séries serão diferenciadas, segundo Montegomery (2009). Para a tomada de decisão na escolha de qual o melhor modelo, frente a todos os modelos testados, foi escolhida a seguinte estratégia: Como primeiro critério foi definida a presença de ruído branco, situação que é caracterizada pela presença de média zero, variância constante e variáveis não correlacionadas. Em seguida foi escolhido o modelo que apresentou os menores valores para os critérios penalizadores, AIC e BIC. Por fim a decisão também foi baseada no critério da parcimônia, situação onde se encontra o menor número de parâmetros, entre os modelos que apresentaram ruído branco e significância de $5 \%$, que resultaram nas estimativas mais precisas.

Para as etapas de enxágüe e de neutralização, estão apresentados na tabela 1 , os seguintes modelos concorrentes:

Tabela 1 - Modelos concorrentes para a variável enxágüe e neutralização de acordo com os critérios estabelecidos

\begin{tabular}{|c|c|c|c|c|c|}
\hline Modelo & Coeficientes & $p$-valor $(p \leq 5 \%)$ & AIC & BIC & $\mathbf{R} / \mathbf{B}$ \\
\hline \multicolumn{6}{|c|}{ AR (1) - Enxágue } \\
\hline Cte & 14076,30 & & & & \\
\hline$\Theta_{1}$ & 0,983397 & 0,0000 & 14,64998 & 14,69547 & Sim \\
\hline \multicolumn{6}{|c|}{ ARMA $(1,1)$ - Enxágue } \\
\hline Cte & 15217,37 & & & & \\
\hline$\Phi_{1}$ & 0,986266 & 0,0000 & & & \\
\hline$\Theta_{1}$ & $-0,211445$ & 0,0197 & 14,62949 & 14,69772 & $\operatorname{sim}$ \\
\hline
\end{tabular}

ARIMA $(0,1,1)$ - Enxágue 


\begin{tabular}{|c|c|c|c|c|c|}
\hline Modelo & Coeficientes & $p$-valor $(p<-5 \%)$ & AIC & BIC & $R / B$ \\
\hline \multicolumn{6}{|c|}{ AR (1) - Neutralização } \\
\hline$\overline{\text { Cte }}$ & 13,41283 & & & & \\
\hline$\Phi_{1}$ & 0,800555 & 0,0000 & $-3,712337$ & 3,66849 & Sim \\
\hline \multicolumn{6}{|c|}{ AR (2) - Neutralização } \\
\hline$\overline{\text { Cte }}$ & 13,41272 & & & & \\
\hline$\Phi_{1}$ & 0,630387 & 0,0000 & & & \\
\hline$\Phi_{2}$ & 0,220844 & 0,0147 & $-3,755714$ & $-3,687124$ & $\operatorname{sim}$ \\
\hline
\end{tabular}

\begin{tabular}{llllll}
\multicolumn{6}{c}{ ARMA $(1,1)$ - Neutralização } \\
\hline Cte & 13,41741 & & \\
$\Phi_{1}$ & 0,913813 & 0,0000 & & \\
$\Theta_{1}$ & $-0,321106$ & 0,0029 & $-3,755679$ & $-3,687446$ & Sim \\
\hline \multicolumn{7}{c}{ AR $(2)$ - Neutralização } \\
\hline$\Phi_{1}$ & 0,700869 & 0,0000 & $-3,717824$ & $-3,672097$ & Sim \\
$\Phi_{2}$ & 0,299180 & 0,0007 & & \\
\hline
\end{tabular}

\begin{tabular}{|c|c|c|c|c|c|}
\hline \multicolumn{6}{|c|}{ ARIMA $(1,1,0)$ - Neutralização } \\
\hline Cte & 0,000538 & & & & \\
\hline$\Phi_{1}$ & $-0,299177$ & 0,0007 & $-3,717860$ & $-3,672133$ & Sim \\
\hline \multicolumn{6}{|c|}{ ARIMA $(0,1,1)$ - Neutralização } \\
\hline$\overline{\mathrm{Cte}}$ & 0,000804 & & & & \\
\hline$\Theta_{1}$ & $-0,399579$ & 0,0000 & $-3,738678$ & $-3,693189$ & Sim \\
\hline \multicolumn{6}{|c|}{ ARIMA $(1,1,0)$ - Neutralização } \\
\hline$\overline{\Phi_{1}}$ & $-0,298736$ & 0,0007 & $-3,733766$ & $-3,710902$ & $\overline{\operatorname{Sim}}$ \\
\hline \multicolumn{6}{|c|}{ ARIMA $(0,1,1)$ - Neutralização } \\
\hline$\overline{\theta_{1}}$ & $-0,397470$ & 0,0000 & $-3,753497$ & $-3,730753$ & $\operatorname{sim}$ \\
\hline
\end{tabular}

Dos modelos concorrentes, acima apresentados, formam selecionados, de acordo com a estratégia acima detalhada, para as etapas de enxágüe e neutralização os seguintes modelos, apresentados na tabela 2 .

Tabela 2 - Modelos selecionados para a variável enxágüe e neutralização de acordo com os critérios estabelecidos

\begin{tabular}{llllll}
\hline Modelo & Coeficientes & p-valor $(\mathbf{p} \leq \mathbf{5 \%})$ & AIC & BIC & R/B \\
\hline Cte & 14076,30 & AR (1) - Enxágue & \\
$\Theta_{1}$ & 0,983397 & 0,0000 & 14,64998 & 14,69547 & Sim \\
\hline \multicolumn{7}{c}{ AR (1) - Neutralização } \\
\hline Cte & 13,41283 & & \\
$\Phi_{1}$ & 0,800555 & 0,0000 & $-3,712337$ & 3,66849 & Sim
\end{tabular}


Fonte: Programa Eviews - versão 8

Os modelos selecionados cumpriram a condição dos resíduos serem ruído branco - RB e não autocorrelacionados.

Nas Fig. 3 e 4, apresenta-se o gráfico da variável original, a ajustada pelo modelo proposto e o comportamento dos resíduos das etapas de enxágüe e neutralização. Pode-se observar que a série original e a modelada apresentam comportamento similar, sinalizando que o modelo adotado foi capaz de captar os movimentos das séries, portanto o modelo foi convergente. Analisando-se os resíduos pode-se verificar um comportamento aleatório, corroborando graficamente a ideia de não autocorrelação dos resíduos e, portanto independente, oscilando em torno de uma média constante próxima de zero. O que caracteriza um modelo que produziu resíduos com características de ruído branco.

Segue abaixo o gráfico onde se verifica um comparativo entre a série original, a série ajustada e o gráfico dos resíduos da modelagem.

Fig. 03 - Gráfico das séries originais, ajust. e resíd. - Enxágue

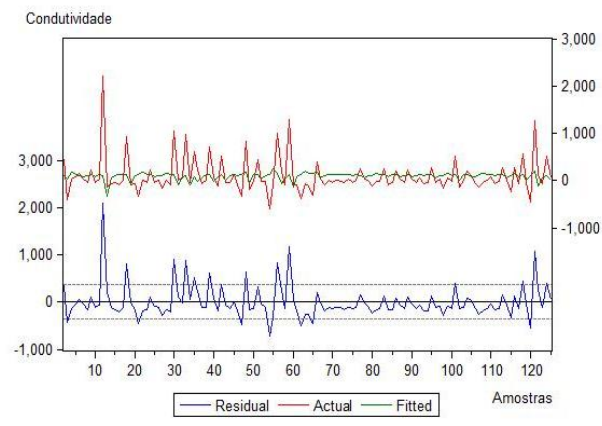

Fonte: Programa Eviews - versão 8
Fig. 4 - Gráfico das séries originais, ajust. e dos res. - Neutral

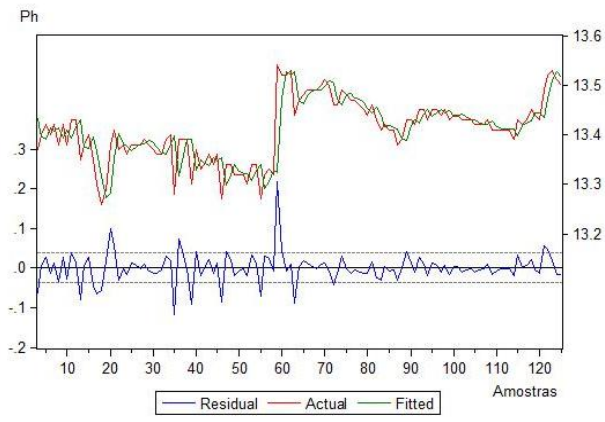

Fonte: Programa Eviews - versão 8

Dado que os resíduos apresentam características de independentes e identicamente distribuídos - i.i.d.,é possível aplicar gráficos de controle.

$\mathrm{Na}$ apresentação seqüencial do trabalho apresentam-se, nas Fig. 5, 6, 7 e 8 os gráficos de controle, conforme orientação do autor citado, sobre os resíduos conseqüentes dos melhores modelos encontrados.
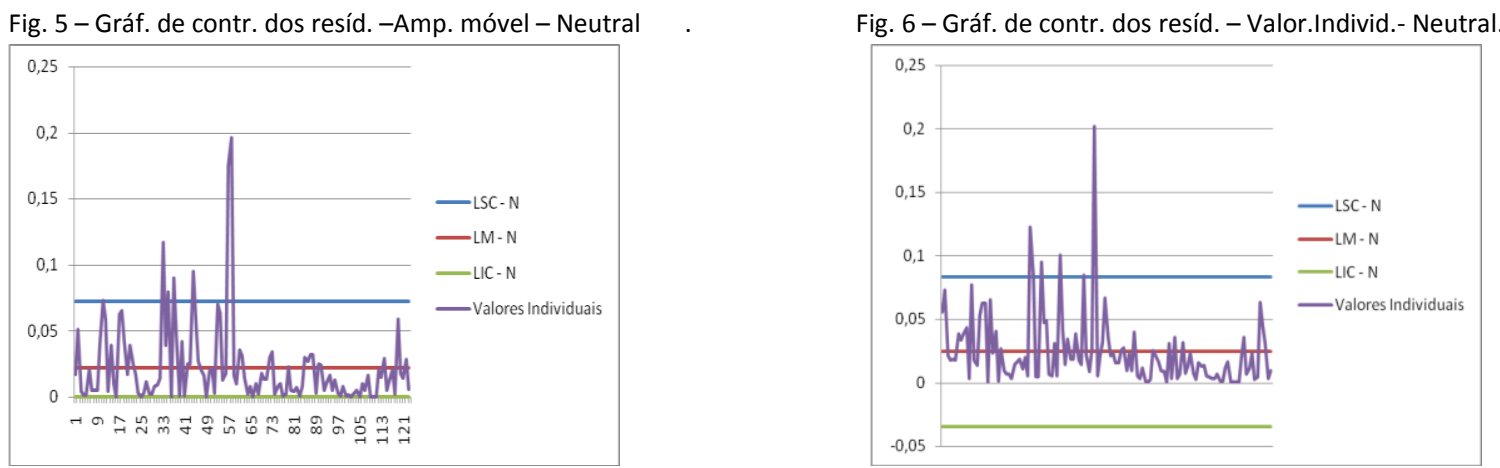

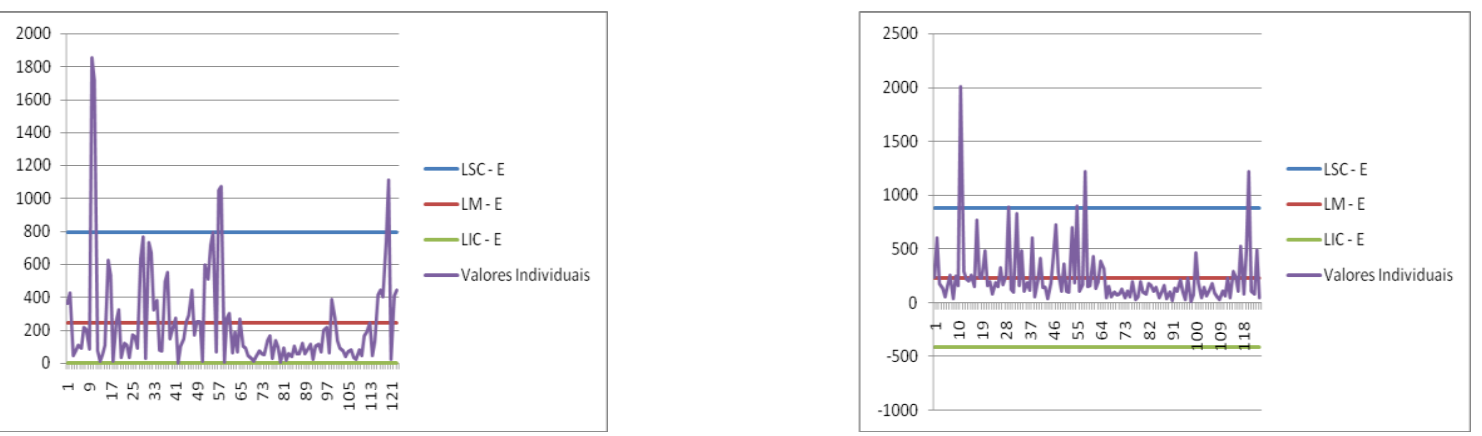

Para o entendimento das sugestões de alteração no processo, primeiramente, frente à análise realizada, deve-se entender que o processo de enxágüe de peças pode ser considerado como uma etapa de lavagem das mesmas, que tem o intuito de retirar todos os resíduos e ou soluções utilizadas nas etapas anteriores. Esse sistema, quanto ao seu tamanho e tipo, é particular de cada empresa e levará em consideração o volume e as dimensões das peças trabalhadas. É oportuno frisar que o sistema utilizado pela empresa nessa etapa é uma lavagem estanque.

Os sistemas de lavagem podem ser de três tipos: lavagem estanque, tanques de lavagem corrente e lavagem em cascata. No tanque de lavagem estanque, sistema onde há um volume de líquido constante, sem entrada ou saída de água, e que por conseqüência, durante o seu uso a concentração do banho aumenta continuamente com o arraste (volume de solução que é aderido à superfície das peças e transportado por esta aos banhos subseqüentes). Já na lavagem estanque combinada com o sistema em cascata, conforme Ponte (2014, p.25) é uma boa solução para melhorar o processo, o volume da água utilizada não é fixo e, dessa maneira, se obtém um menor arraste de particulados, somado a um menor consumo de água. A partir do exposto acima pelo autor, e analisando os gráficos gerados, verifica-se como alternativa para um controle mais rigoroso uma mudança no processo, fazendo o enxágüe das peças utilizarem esse sistema, onde como consequiência, haverá uma diminuição da variabilidade das medidas capturadas, tendo como resultado um estado de controle do processo.

Ainda como detalhe importante para o momento da coleta de dados e também como justificativa aos pontos encontrados fora dos limites de controle no gráfico dos resíduos, verifica-se como explicação uma coincidência entre o instante da captura das amostras com a entrada e ou saída das peças do tanque de processo, momento este que gera uma movimentação extra da água de processo que geraram amostras que podem ter influenciado os pontos extremos.

\section{CONCLUSÃO E PERSPECTIVAS DE TRABALHOS FUTUROS}

A pesquisa mostrou a importância da modelagem ARIMA no suporte à tomada de decisões. Inicialmente a ideia central estava baseada na necessidade de um melhor controle do processo galvânico na etapa estudada. A alternativa de um reestudo do processo, que resultou na hipótese de instalação de um sistema de lavagem em forma de cascata na etapa de enxágüe, onde diminuiria o araste dos contaminantes para as etapas seguintes do processo, só foi tomada a partir dos gráficos gerados com os resíduos da modelagem ARIMA que mostraram pontos fora dos limites de controle.

A adoção do sistema proposto acima ainda teria como benefício um menor consumo de água, matéria-prima utilizada em grande quantidade nessa operação, o que vai ao encontro de um sistema produtivo ambientalmente correto. 
O processo de galvanização eletrolítica, apresentado em detalhes no texto visto anteriormente, é composto por várias etapas, apresentando correlação e autocorrelação entre todas as fases. Frente a esta realidade, deixa-se como sugestão um estudo que avalie as seqüências de operações anteriores e posteriores a estudada, visando maximizar e ao mesmo tempo diminuir a variabilidade do processo como um todo.

$\mathrm{O}$ estudo tem como sugestão de pesquisas futuras a aplicação de uma modelagem ARMAX de modo que uma variável exógena possa ser utilizada para explicar as variáveis envolvidas no processo. Assim como também se busca a investigação de efeitos de volatilidade por meio da modelagem ARCH- autoregressivo condicional a heterocedasticidade.

Também com o acréscimo de estudos, como a modelagem ARMAX, busca-se resultados que apontem para o exato momento em que o processo entra em descontrole.

\section{REFERÊNCIAS BIBLIOGRÁFICAS}

CAI, D.Q.; XIE, M.; GOH, T.N.; TANG, X.Y. Economic design of control chart for trended processes. International journal of production economics; 85-92 p.; abr. 2002.

CASARIN, V. A.; KLIDZIO, R.; SOUZA, A. M.; SPIM, J. A. Application of ar-arch model in the monitoring of autoregressive processes through residues control charts. Revista Espacios, Vol. 34, abr.2013.

COSTA, A. F. B.; EPPRECHT, E. K.; CARPINETTI, L. C. R. Controle estatístico de qualidade. $2^{\text {a }}$ ed.São Paulo: Atlas, 2012.

DICKEY, D. and FULLER, W. Distribution of the estimators for autoregressive time series with a unit root. Journal of the American Statistical Association, v. 84, 427-431p.1979.

ENDERS, W. Applied econometric time series. Wiley series in probability and mathematical statistics. John Wiley and Sons, Inc., New York. N.Y.1995.

KWIATKOWSKI, D.; PHILLIPS, P. C. B.; SCHMIDT, P.; SHIN, Y. Testing the null hypothesis of stationarity against the alternative of a unit root. Journal of Econometrics, 54. 1992.

LEE, Y.; TONG, L.Forecasting time series using a methodology based on autoregressive integrated moving average and genetic programming. Knowledge-Based Systems; 66-72 p.; jul.2010.

MADDALA, G.S. Introduction to econometrics. $2^{\text {a }}$ ed. Prentice-Hall Inc. Englewood Cliffs, New Jersey. 1992.

MINGOTI, S. A; YASSUKAWA, F. R. S. Uma comparação de gráficos de controle para a média de processos autocorrelacionados. Revista eletrônica Sistemas e Gestão, v. 3; n. 1; 55-73 p.; abr. 2008.

MONTGOMERY, D.C. Introdução ao controle estatístico de qualidade. $4^{\mathrm{a}}$ ed. Rio de Janeiro: LTC,2009.

MORRETIN, P. A.; TOLOI, C.M.C -Previsão de séries temporais. $2^{a}$ ed. São Paulo: Edgard Blücher, 2006. 
OLIVEIRA, S. C.; PEREIRA, L. M. M.; HANASHIRO, J. T. S.; VAL, P. C. A study about the performance of time series models for the analysis of agricultural prices. GEPROS Gestão da Produção, operaç̃̃es e sistemas; ano 7; n.3; 11-27 p.; jul-set.2012.

OTERO, U. B.; ROSENFELD, S.; GADELHA, A. J. Óbitos por desnutrição em idosos, São Paulo e Rio de Janeiro. Análise de séries temporais. 1980-1986. Revista Brasileira de Epidemiologia; vol. 4, n 3, 191-205 p. 2001.

PEREIRA, Z. P.; REQUEIJO, J. G. Qualidade: Planeamento e controlo estatístico de processos. Ed. Prefácio edição de livros e revistas Ltda: Lisboa.2008.

PONTE, H. A. Tratamento de efluentes líquidos de galvanoplastia. Disciplina: Eletroquímica aplicada e corrosão - TQ - 417. UFPR- Universidade Federal do Paraná- Setor de tecnologia - Departamento de engenharia Química.

ROTONDARO, R. G. Seis sigma: estratégia gerencial para a melhoria de processo, produtos e serviços. São Paulo: Atlas, 2002.

SOUZA, A. M.; SOUZA, F. M.; FERREIRA, N.; MENEZES, R. Eletrical energy supply for Rio Grande do Sul, Brazil, using forecast combination of weighted eigenvalues. GEPROS Gestão da Produção, operações e sistemas; n 3, 23-39 p.; jul-set. 2011.

SOUZA, A.M.; SOUZA, F.M.; MENEZES, R.Procedure to evaluate multivariate statistical process control using ARIMA-ARCH models. Japan Industrial Management Association; vol. 63; n 2E; 112-123 p.; abr. 2012.

WOODALL, W. H.Controversies and contradictions in statistical process control. Journal of Quality Technology. V. 32; n.4; 341-350 p.; October.2000.

ZEMPULSKI, L. N.; ZEMPULSKI, M. F. S. Instituto de Tecnologia do Paraná; Dossiê Técnico. Galvanização Eletrolítica. 2007. 